\title{
Implementation of Low Power and Area Efficient Vedic Multiplier
}

\author{
Shobana Priya M S, Manikandan T, Joshua Kumaresan S, Satheesh Kumar S, Muruganandham A
}

\begin{abstract}
Designing a low power consuming and area efficient Vedic Multiplier using Hybrid Full Adder. In this paper, Conventional CMOS (CCMOS) Full Adders involved in a conventional Vedic multiplier is replaced with Hybrid Full adders to achieve reduction in power consumption and area. In the proposed system ripple carry adders involved in Vedic multiplier are designed using Hybrid Full Adder. The design is done for 2-bit and it is extrapolated to 16-bit. Performance parameters such as power consumed and area between Vedic multiplier involving CCMOS and Hybrid Full Adder is done and a comparative study over them is made. Significant improvement is achieved in this implementation and the layout design is also implemented for the 2-bit, 4-bit, 8-bit and 16-bit Vedic multiplier for both Conventional CMOS and Hybrid Full-Adder logic styles. The implementation is carried out using Tanner EDA tool under 250-nm technology.
\end{abstract}

Keywords: Multiplier, CMOS, Full Adder, Low Power, Small Area.

\section{INTRODUCTION}

Arithmetic operations plays a vital role in many real-time applications. Specifically, for VLSI application it is the main building block. To improve the performance of the application, optimization of multipliers is carried out using adders. A multiplier is the main block in various applications such as Digital Signal Processing, Cryptography for the process of encryption and decryption and in other logics. Many experiments have been conducted to obtain optimized Multipliers which is Area, Power and Delay efficient. Vedic Multiplier is said to have may advantages over other multipliers.

The well-known and the basic structure of Vedic Multiplier used in almost many Digital Signal Processing system and they are designed with the complementary metal oxide semi-conductor. It has many Half-Adders and Full-Adders which leads to power dissipation and delay to the overall circuit. The delay and power optimization of the

Revised Manuscript Received on November 12, 2019

Ms.M.S.Shobana Priya, M.E.,(Ph.D), Assistant Professor, Department of ECE, Saveetha School of Engineering, Mail id: shobanapriyams.sse@saveetha.com

Dr. T.Manikandan, Professor, Department of ECE, Rajalakshmi Engineering College, Mail id: manikandan.t@rajalakshmi.edu.in

Dr.S.Joshua kumaresan, Associate Professor, Department of ECE, R.M.K. Engineering College, skn.ece@rmkec.ac.in Krishna College of Engineering and Technology, ssatheeshkumarpsg@gmail.com

Dr. A. Muruganandham, Professor, Department of ECE, Rajarajeswari college of Engineering, Mail id: a.muruganandham@gmail.com
Mr. S. Satheesh Kumar, Assistant Professor, Department of ECE, Sri

circuit is carried out by reducing the number of transistors employed in the basic adder level design. The conventional CMOS Full-Adder are replaced using Hybrid Full-Adders is used in this paper. The performance of these modified implementations is compared with those containing Conventional CMOS Full-adders and optimization with respective to area and power consumption is achieved in the proposed design.

\section{Vedíc Multiplier[6]}

Vedic Mathematics forms the basis for Vedic Multiplier. Vedic Mathematics is a method of processing with efficient and speedy implementation of digital circuits with set of arithmetic rules Vedic Multiplier has an advantage of faster computation. Vedic Multiplier functions based on the Sutras, that are the Vedic multiplication formulas. There are 16 sutras present in order to carry out with the multiplication operation. The "Urdhva Tiryakbhyam" sutra (algorithm) is used in the proposed Vedic Multiplier. This multiplier was traditionally used in the multiplication of numbers in decimal number system. This formula is a general multiplication technique which forms the basis for all sorts of multiplication. The block diagram of n-bit Vedic Multiplier is given in Fig 1.

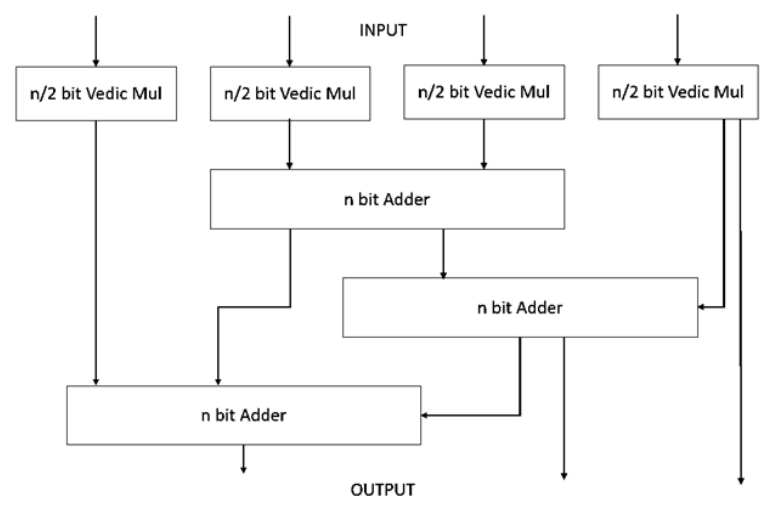

Fig. 1. n-bit Vedic Multiplier

The multiplier uses the algorithm Urdhva Tiryakbhyam (Vertical \& Crosswise) of antique Vedic Mathematics. The conceptual meaning of Urdhva Tiryakbhyam is "perpendicularly and diagonally". The working of the algorithm is with the calculation of partial products. Simultaneous addition of partial products are carried out with this. The example of two numbers with this method is given in Fig 2. 


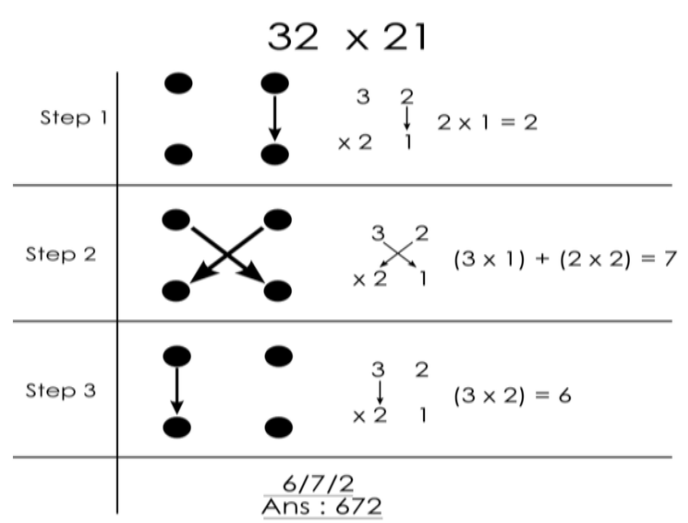

Fig 2. Model Calculation of Vedic Multiplier.

The 16-bit Vedic Multiplier structure consist of four 8-bit Vedic Multipliers and three 16-bit Ripple Carry Adders. The 8-bit Vedic Multiplier needs four 4-bit Vedic multipliers and three 8-bit Ripple Carry Adders. The 4-bit Vedic multiplier needs four 2-bit Vedic multiplier and three 4-bit Ripple Carry Adders. The structure of 16-bit Vedic multiplier is given in Fig 3.

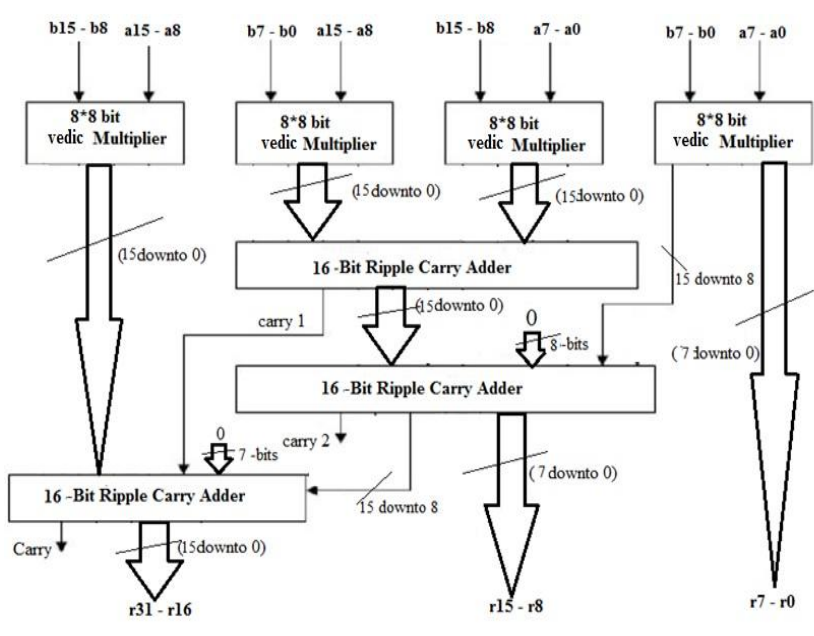

Fig 3. 16 bit Vedic Multiplier circuit.

\section{HYBrid FULL-AdDER [5]}

The hybrid logic has a module for carry generation using modified XNOR. In hybrid logic (small c) conventional CMOS(CCMOS) and transmission gates are coupled together to obtain low power and high speed operation. The XNOR module is designed with Conventional CMOS and the module to generate carry is designed using transmission gates. Fig 4 shows the block diagram of Hybrid Full adder. There are two modules that are present in the design of Hybrid Full-Adder. One module is the Carry/Sum Generation Module and the other Module is the XNOR module. To achieve faster conduction, the Weak inverter is used. The weak inverter consists of smaller depletion layer that helps in quicker conduction.

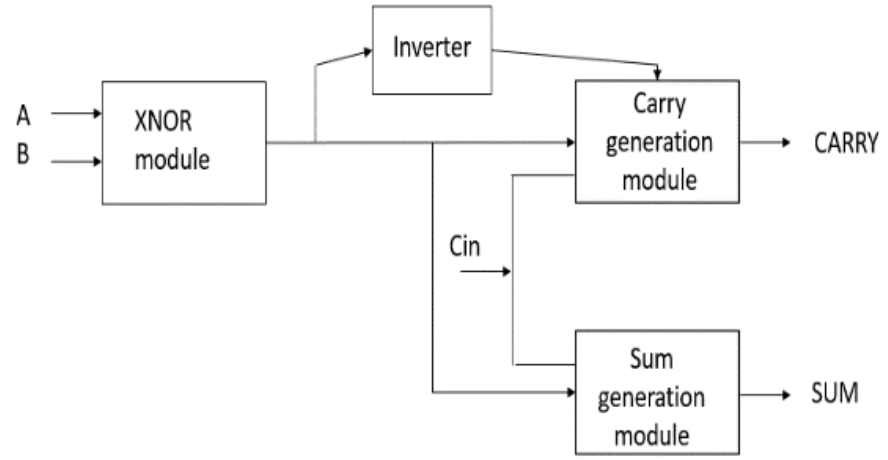

Fig 4. Hybrid Full-Adder

Fig 5 shows the transistor level diagram of Hybrid Full adder.

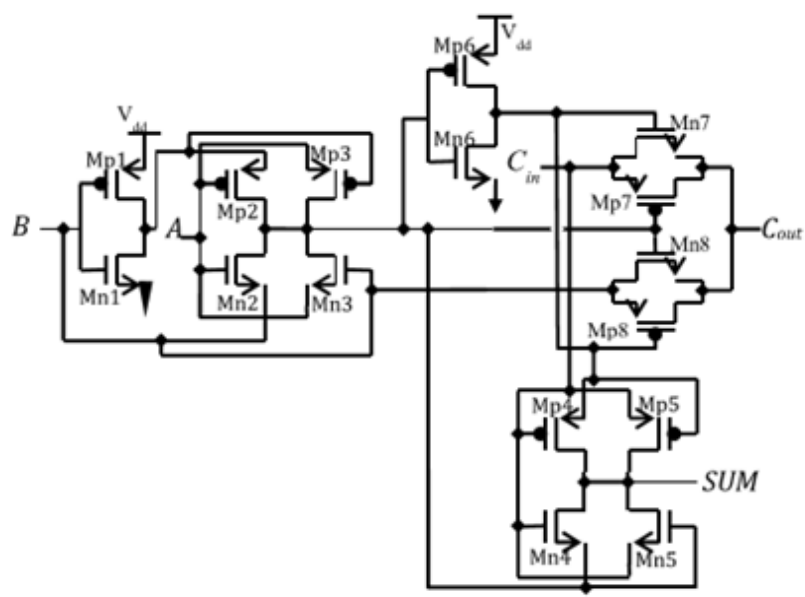

Fig 5. Hybrid Full-Adder (Transistor level)

In the place of normal Full-Adders, these Hybrid Full-Adders are used in Ripple Carry Adders of the Vedic Multiplier.

\section{RESUlts AND Discussion}

The implementation of 16-bit Vedic Multiplier using CCMOS and Hybrid Full adders in Carry Save Adder block is carried out in Tanner EDA tool. The Area and Power Consumption calculations are done in in $180 \mathrm{~nm}$ Technology. Figs 6 shows the schematics of the Multiplier design.

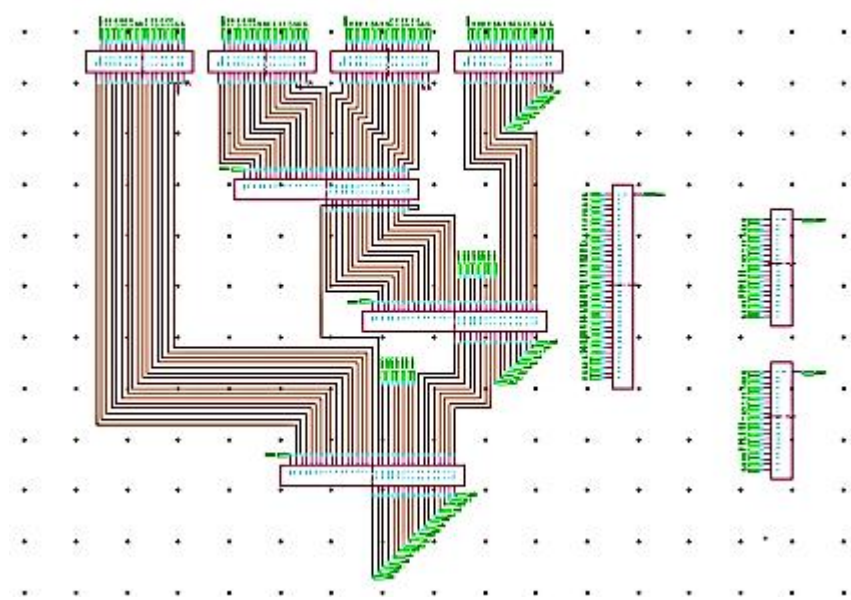

Fig 6. Schematic of Vedic Multiplier. 
The Output of the 16-bit design is given in Fig 7. The Layout design is also done or the 2-bit, 4-bit, 8-bit and 16-bit Vedic Multipliers using both CCMOS and Hybrid Full-Adders. The Layout of 16-bit Vedic Multiplier using CCMOS and Hybrid Full-Adders is given in Fig 8 and Fig 9.

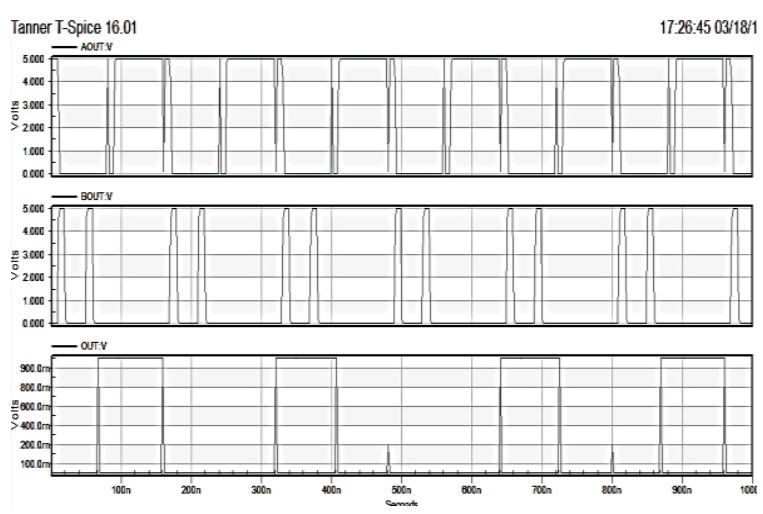

Fig 7. Output of 16-bit Vedic Multiplier

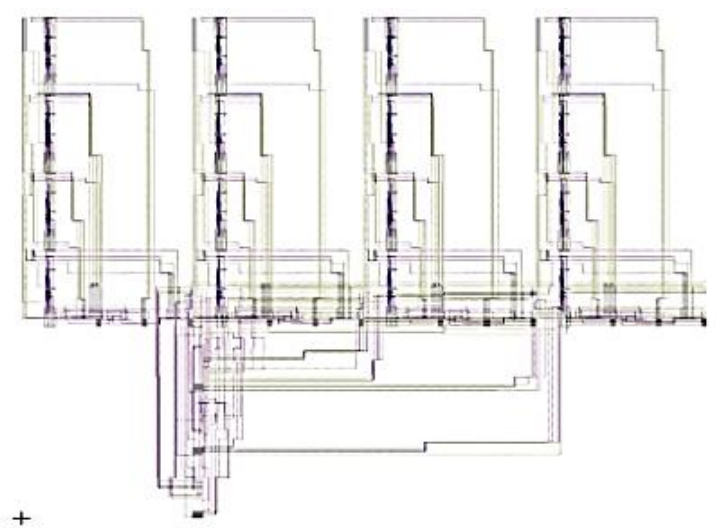

Fig 8. Layout of 16-bit Vedic Multiplier using CCMOS Full-Adder

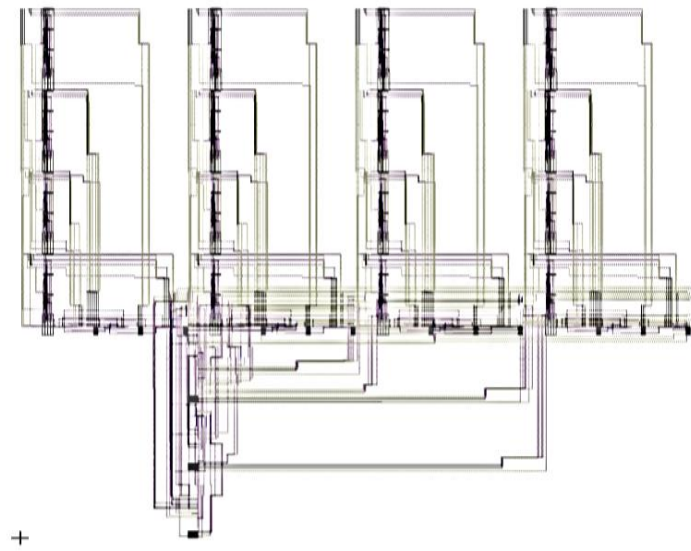

Fig 9. Layout of 16-bit Vedic Multiplier using Hybrid Full-Adder

The performance comparison of 16-bit Vedic Multiplier when executed by replacing Conventional Full-Adder with Hybrid Full-Adder has optimizes Area, Power Consumption. The bit size occupies are 2-bit, 4-bit, 8-bit and 16-bit. Table 1 shows the area and power Consumption of Vedic Multiplier with CCMOS and Hybrid Full-adders.
Table 1. Area and Power Consumption of Vedic Multiplier with CCMOS and Hybrid Full adders

\begin{tabular}{|c|l|c|c|c|}
\hline $\begin{array}{c}\text { Multiplier bit } \\
\text { size }\end{array}$ & \multicolumn{3}{|c|}{ AREA } & \multicolumn{2}{c|}{ POWER(watts) } \\
\cline { 2 - 5 } & CCMOS & HYBRID & CCMOS & HYBRID \\
\hline 2-bit & 58 & 58 & $3.1678 \mathrm{e}-006$ & $4.1566 \mathrm{e}-007$ \\
\hline 4-bit & 568 & 424 & $6.9876 \mathrm{e}-006$ & $8.14567 \mathrm{e}-007$ \\
\hline 8-bit & 2944 & 2080 & $1.23789 \mathrm{e}-005$ & $2.83456 \mathrm{e}-006$ \\
\hline 16-bit & 13900 & 9868 & $2.9876 \mathrm{e}-004$ & $4.56788 \mathrm{e}-005$ \\
\hline
\end{tabular}

\section{GRAPHiCAL REPRESENTATION}

Figs 10 and Fig 11 show the graphical representation of the result obtained in table 1

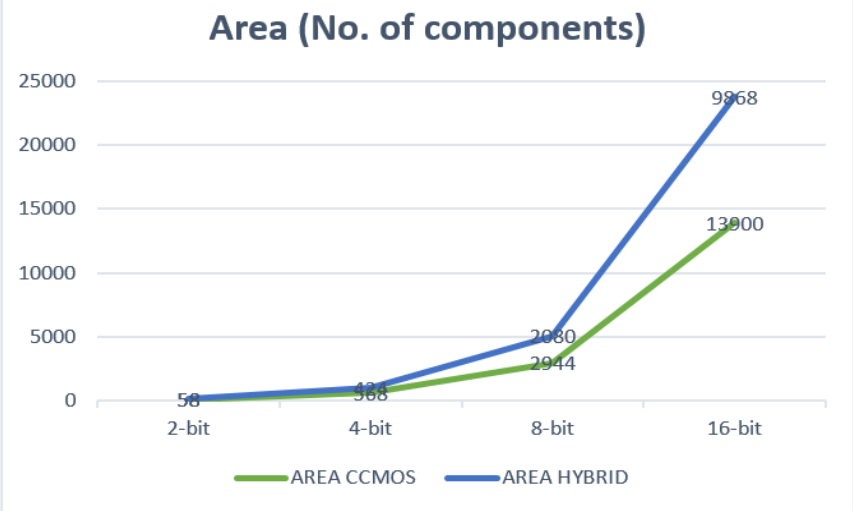

Fig 10. Area Comparison

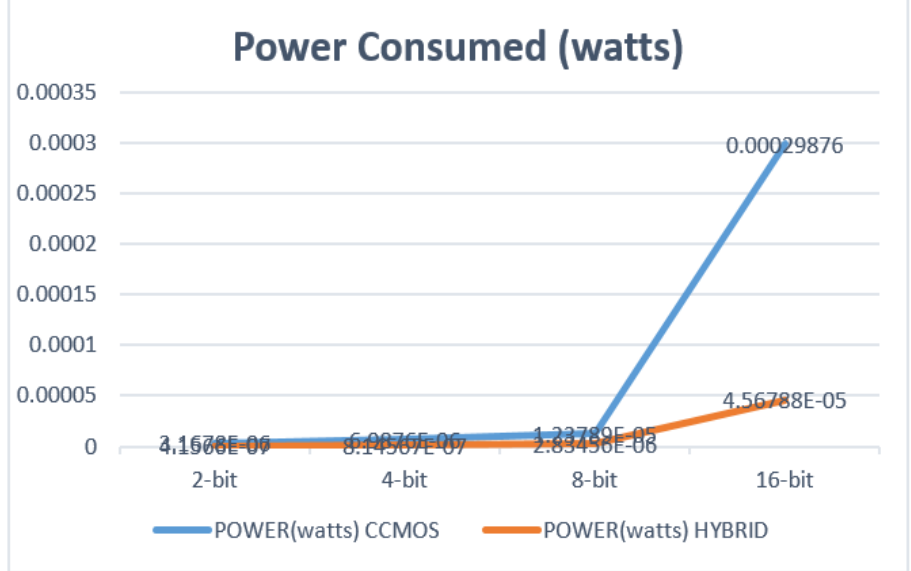

Fig 11. Power Consumption Comparison (in watts)

\section{Conclusion}

In this paper, Analysis of Low-Power consuming Area Efficient Vedic Multiplier (16-bit) is done using Hybrid Full-Adder Circuit is proposed, designed and simulated in Mentor Graphics Tanner EDA tool. The output of the Multiplier 
designed with proposed Hybrid logic is compared with the existing logic styles of Full-adders in 16 bit. The 2-bit, 4-bit, 8-bit and 16-bit Vedic Multipliers are designed in both Conventional CMOS and Hybrid Full-Adder logic styles.

From the simulated result using Tanner EDA tool the Power consumption of the existing logic styles are reduced by the proposed design by $87 \%$ for 2-bit, $89 \%$ for 4 -bit, $78 \%$ for 8 -bit and $85 \%$ for 16-bit when compared with the existing logic of C-CMOS to Hybrid Full-Adder and also the area (number of components) is also reduced by $36 \%$ for 4-bit, $30 \%$ for 8 -bit and $30 \%$ for 16-bit when compared with C-CMOS logic to Hybrid Full-Adder logic design. The layout design is also implemented for the 2-bit, 4-bit, 8-bit and 16-bit Vedic multiplier for both Conventional CMOS and Hybrid Full-Adder logic styles.

\section{ACKNOWLEDGMENT}

The authors would like to thank College of the author and the co-authors for providing the required facility in the research work.

\section{REFERENCES}

1. J. M. Rabaey, A. Chandrakasan, and B. Nikolic, Digital Integrated Circuits: A Design Perspective, 2nd ed. Delhi, India: Pearson Education, 2003.

2. N. H. E. Weste, D. Harris, and A. Banerjee, CMOS VLSI Design: A Circuits and Systems Perspective, 3rd ed. Delhi, India: Pearson Education, 2006.

3. K. Navi, M. Maeen, V. Foroutan, S. Timarchi, and O. Kavehei, "A novel low-power full-adder cell for low voltage,” VLSI J. Integr., vol. 42, no. 4 , pp. 457-467, Sep. 2009.

4. Pushpalata Verma, "Design of $4 \times 4$ bit Vedic Multiplier using EDA Tool," Inter-national Journal of Computer Applications (0975 - 888)., vol. 48 , No.20, pp. 32-35, June 2012

5. P. Bhattacharyya, B. Kundu, S. Ghosh, V. Kumar and A. Dandapat, "Perfor-mance Analysis of a Low-Power High-Speed Hybrid 1-bit Full Adder Circuit,” IEEE Trans. Very Large Scale Integr. (VLSI) Syst., vol. 23, no. 10, pp. 2001-2008, Oct. 2015.

6. P.Kamila, Parveen and C.Priya, "Multiplier Design using MTCMOS with Adap-tive Hold Logic," International Conference on Advanced Communication Control and Computing Technologies (ICACCCT) 2016.

7. JP. L. Montgomery, "Modular multiplication without trial division," Math. Com-put., vol. 44, no. 170, pp. 519-521, Apr. 1985.

\section{AUTHORS PROFILE}

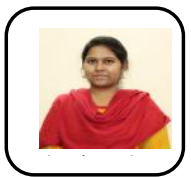

Mrs. M S. Shobana Priya is a Assistant Professor working at Saveetha School of Engineering, Thandalam, Chennai. She is currently pursuing her Ph.D in Machine learning at Saveetha School of Engineering, SIMATS, Thandalam since June, 2018. She did her bachelor's degree in Engineering from Jaya Engineering College, Thiruniravur under Anna University \& master's degree in Engineering from College of Engineering, Anna University, Guindy during 2011 \& 2015, respectively. She is having 1.5 years of teaching experience in UG. She also guided many projects at UG levels.

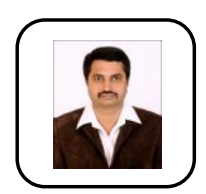

Dr. T. Manikandan is a Professor working at Rajalakshmi Engineering College, Thandalam, Chennai. He has completed his Ph.D titled "A study on computer-aided diagnosis systems for lung cancer detection and its three dimensional visualization using machine learning techniques" at Anna University, Chennai during January,

2017. He did his bachelor's degree in Engineering from Vellore Engineering College, Chennai under Madras University \& master's degree in Engineering from College of Engineering, Anna University, Guindy, Chennai during 1998 $\& 2007$, respectively. He is having 21 years of teaching experience which includes both UG and PG. He also guided many projects at UG \& PG levels. He has 50 papers in refereed international journals and conferences in his credit. $\mathrm{He}$ is serving as editorial board member for various international journals such as International journal of Biomedical and Healthcare Sciences, Archives of
General Internal Medicine, Journal of Cancer Diagnosis, Journal of Medical and Clinical Oncology, Journal of Clinical Epigenetics and Integrative Cancer Biology and Research.



Dr.S.Joshua Kumaresan is working as Associate professor in the department of ECE of R.M.K. Engineering college. He completed his $\mathrm{Ph} . \mathrm{D}$ in the area of image processing in 2018, M.E (VLSI Design) in 2007 both from Anna university Chennai. He also completed M.S (Electronics and Control) from BITS, Pilani in 1999 and B.E (ECE) in 1994 from Bharathidasan University. He has 23 years of teaching experience which includes both UG and PG. and 1 year he worked in an industry as R\& D Engineer. He published more than 20 papers in international journal and conferences.He published books on Electromagnetic Fields, Digital Electronics, Digital Principles system design and Digital logic circuits. . He guided many projects at UG \& PG levels. He also mentored and guided many students for various competitions like Smart India Hackathon, Arm design contest, India Innovation challenge design contest 2018 etc. He organized many college level National conferences and symposiums. He is a member of professional societies IEI, ISTE, ISRO-ISSE, and IACSIT.

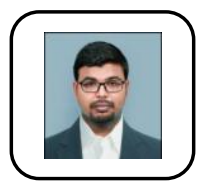

Satheesh Kumar S currently working as Assistant Professor at Sri Krishna College of Engineering and Technology, Coimbatore. He accomplished his Master of Engineering in the field of Communication Systems from PSG College of Technology, Coimbatore and B.E. from Anna University, Chennai. He is also a Life time member of IETE and ISRD. Having more than 4 years of teaching experience, he is acting as a board member for some Private Engineering Institutions in Coimbatore. He is also a recipient of various honors and faculty awards in his teaching fraternity. He also published over 20 technical articles in various International and national journals and filed a patents in his research field.His research focus include Electromagnetics, Microwave Engineering, Speech Processing and Block Chain technology.

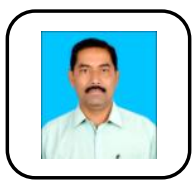

Dr. A. Muruganandham received the B.E. Degree in Electronics and Communication Engineering and M.E., degree in Communication systems from University of Madras and National Institute of Technology (NIT), Trichy in 1993 and 2000, respectively. He completed the $\mathrm{Ph} . \mathrm{D}$ (Image Compression) degree in Anna University in the year 2013, Chennai. He is a life member of ISTE, FIEand member of IEEE. $\mathrm{He}$ is currently working as a Professor in the Department of Electronics and Communication Engineering, at Rajarajeswari College of Engineering, Bengaluru, Karnataka, India and has a teaching experience of 23 years His-research interests includes Image processing and Bio Medical Engineering. 